\title{
Efficient Broadcasting Protocol for Vehicular Ad-Hoc Network
}

\author{
Mahipal \\ Computer Science Department \\ PEC University of Technology \\ Sector-12, Chandigarh
}

\author{
Sanjay Batish \\ Computer Science Department \\ PEC University of Technology \\ Sector-12, Chandigarh
}

\author{
Arvind Kakria \\ Computer Science Department \\ PEC University of Technology \\ Sector-12, Chandigarh
}

\begin{abstract}
The Vehicular Ad-hoc Networks is one of the most challenging research areas in the field of Mobile Ad Hoc Networks. In the VANET most of the messages sent through broadcasting. In VANET connection between two nodes break due to the high mobility of vehicles. So the broadcasting of messages in vehicular ad-hoc network is still an open research challenge and requires some efforts to reach an optimum solution. In this paper, we addressed exactly this problem. We propose a flexible, simple, and scalable design for VANET and new protocol for broadcasting which broadcast the message in an efficient way and also reduce channel overhead, and make Better packet reception rate
\end{abstract}

\section{General Terms}

Broadcasting Protocol for VANET.

\section{Keywords}

VANET, Broadcast, Unicast, ACK, GPS, RTS, CTS, Contention window.

\section{INTRODUCTION}

Broadcasting of the messages will play a greater role in VANET than unicasting. The most of the messages sent in the network are broadcast in nature. The basic uses of broadcasting to broadcast emergency warning messages and vehicles state like a vehicles velocity, acceleration, location, and direction. To achieve reliability and efficiency of broadcast messages in a VANET provide many technical problem. In vehicular ad-hoc network the retransmission of failed broadcast message is not possible because they are undetectable. But we can detect the failed unicast message. Because we use the acknowledgment (ACK) which is send by the receiver. However, it is not possible to receive an ACK from each node in a broadcast message. If we use ACK, a problem known as the "ACK explosion problem" [1] would exist. Each receiving node would, send the ACK to the transmitting node at almost the same instance; causing collisions. The collision of broadcast message became higher as the distance from the sender increases. Probability of the receiving of broadcast message instantly decreases at distance greater than $66 \%$ of the transmission range [2]. The main factor to decrease the reception rate broadcast message is the hidden terminal problem. Typically, selection of the next relaying hop is the major problem in VANET broadcasting. To get the smallest propagation delay, the number of relaying hops must be minimizing.

Many authors have tried to solve the problem of sending broadcast messages in a VANET. Xu et al. [3] propose a single-hop broadcast protocol that increases the probability of a message's reception by sending the message multiple times. Yang et al. [4] design the new routing protocol named VCWC to transmit the emergency warning messages (EWM), which is based on a state machine and a multiplicative rate decrease algorithm. Both [3] and [4] aim at increasing the probability of reception by broadcasting a message multiple times.

In this paper, we are concerned with exactly this basic problem: we present new protocol in which we propose that how to find the next relaying node in broadcasting and design a method to improvement in the reliability and performance of the network. We implement our propose work on ns2 a network simulator which shows that our protocol improve performance and reduce the no of collision and packet loss.

\section{RELATED WORK}

There are many works regarding VANET broadcast issue. Many of which adapt different Strategies which fall into various categories including distance based, location based, probability based and topology based.

\subsection{Distance based broadcasting}

The distance based approach is the milestone solution for broadcasting. In this approach the node which has greatest distance shall be the next relaying hop. To implement this method, each node set its own timer. When nodes receiving an accident report, they will start the timer. The duration of the timer is inversely proportional to the relative distance between itself and the broadcaster. When the timer is expired, it will relay the message. To overcome the redundant messages, if a node hears the same message twice, meaning that other nodes have already relayed the report. So it will stop its attempt. In this node with the furthest distance to the broadcaster shall be the next relaying hop because its transmission waiting time is the shortest. With this idea, we can achieve lowest message propagation delay due to the minimum number of hops used. However, the reception probability is a potential problem when using this approach because of the large distance [5].

\subsection{Location based broadcasting}

Location based approach is also used in many broadcasting. It is very similar to distance based. The difference is that we use the location obtained from Global Position Devices (GPS) to get the area, map and other useful information as inputs to choose the next relaying hops $[5,6,7,8,9,10]$.

\subsection{Probability based broadcasting}

Probability is usually used to reduce chances of collision and number of transmitted messages. That idea is also applied to VANET [10, 11]. In the broadcasting many used the probability to reduce the transmission chance. Probability can be given by static no or by adaptive no. While some of the works used the fixed static number [11], many of which successfully adapted the adaptive number [10]. Weight $\mathrm{p}$ persistence [11] is the fundamental idea of probability usage in VANET. When message received by some node, node will retransmit with probability $\mathrm{p}$ without using delay timer. Since the vehicle with further distance should have higher priority. Thus, the retransmit probability is proportional to the distance with respect to the broadcaster. Therefore, there is a higher 
chance that node whose distance to broadcaster is greater will relay the message [5].

\subsection{Topology based broadcasting}

Topology based approach is mainly used in wireless sensor network. In VANET topology is change very rapid. So most of the time, topology solution is failed. However, in some scenarios such as freeway where the road is straightforward, it can still be useful. Bononi's [12] used topology based approach in their work in which all nodes creates the backbone topology. The selecting of backbone nodes are based on speed and distance of each node. The backbone nodes will be given responsibility of relaying. Since the topology is created and the relaying path has been established, there will be no contention or collision. However, the process of retaining and creating the backbone is always expensive and sophisticated in high velocity network such as VANET. Fig 1 below shows the vehicle topology.

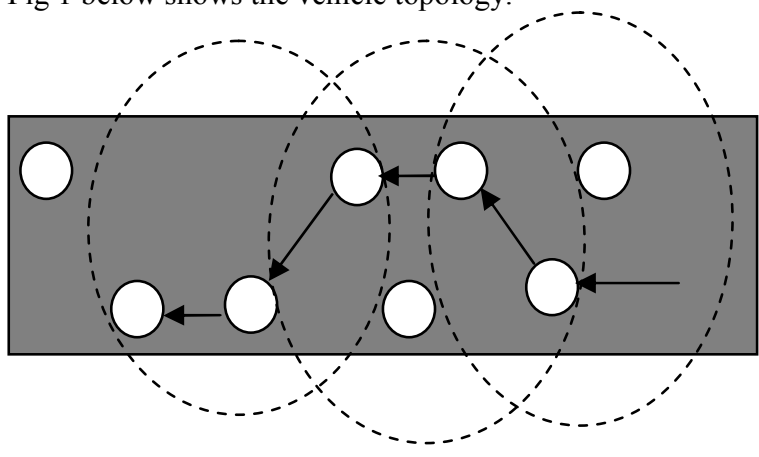

Fig 1: Vehicle topology

\section{PROPOSED WORK}

In this section, we have provided the solutions for weaknesses described in previous section. These solutions can be considered as an efficient broadcasting protocol named EBP (efficient broadcasting protocol). We have also solved the problem of reliability, quality of service and many issue discussed in previous chapter. In the next chapter, we have done performance analysis of both these protocols and the improved protocol. This performance analysis is based on throughput, end to end delay and efficiency.

\subsection{Selection of next relying node}

In our propose method we used beacon message to find the next relaying node. Each node's location information embedded in its beacon message, each node will have knowledge of its neighbors. This neighbor information is very useful for retrieving many useful data such as vehicle density, link reliability, transmission radius etc. In our propose work information broadcasted in the minimum hop to maximum distance. In our method each node calculates the no of nodes covered by their transmission range except those nodes which is covered by the broadcaster.

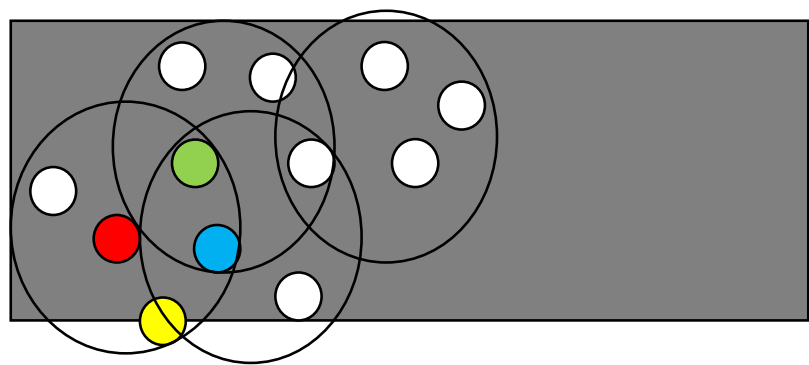

Fig 2: Selection of next relying node
As shown in fig 2 red node is the initiator of the broadcasting. It will broadcast the message to four nodes now who is the next broadcaster is decided base on the no of node covered by that node except those nodes which are covered by red node. In this scenario distance base method fails because three nodes blue, green and yellow are at equal distance from red so it very difficult to find the next relying node. In our propose work we remove such type of draw bags. According to our method green node will be selected because it has higher no node in its transmission range.

\subsection{Broadcast in sparse area}

The broadcasting in VANET is based on the number of vehicles along the path. If there are no more vehicles in the propagation path, the report must be stopped at the point. In broadcasting, a message is relayed by vehicles moving in the same direction as the message initiator. If there is no vehicle in the same direction, the propagation stops. But using beacon message we can send the information in the sparse area. As we state that beacon message also contain the direction of the each node. However, in a bi-directional road, we can use nodes in opposite direction as a relaying node. This so called "messenger" will store a message and carry it while traveling in the opposite direction. The message will be released once it found node in the opposite lane.

\subsection{Contention window}

Apart from the minimizing number of relaying hops, the broadcast reliability is also a major issue. Since RTS/CTS cannot be applied to broadcasting, the problem becomes a serious topic. As in VANET there is no provision to detect collision. So we used a method in which each packet has a sequence no. Each node maintains a broadcast table which contain MAC address, Sequence no, and Time Stamp as shown in the table.

Table 1: Data Maintained in a Broadcast Table

\begin{tabular}{|l|l|l|}
\hline MAC Address & Sequence Number & Time Stamp \\
\hline
\end{tabular}

When a node receive the packet it will match the sequence no and if there any packet loss occurs it will wait for time stamps and report about the packet loss.

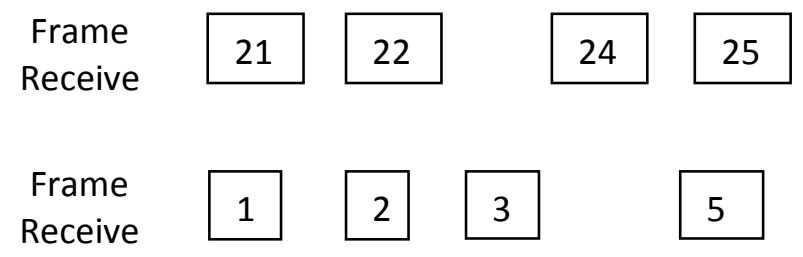

Fig 3: Broadcast Frames Received

As shown in the fig 3 frame 23 and 4 is lost .Now broadcaster can increase the size of contention window so that no of collision reduce. The size of the contention window may either increase or decrease. In our protocol we design an algorithm to change the size of contention window. The algorithm exponential increases the window size as:

$\mathrm{CW}$ new $=2 * \mathrm{CW}$ old +1

For example, if a node is initially transmitting with a $\mathrm{CW}$ equal to 12 and the algorithm determines that the window must be increased, then the new $\mathrm{CW}$ is 25 . The node will then 
randomly set a back off in the range of $[0,25]$. If, when the next Local Reception Rate is calculated, it is determined that the window must be increased again, the new value for the $\mathrm{CW}$ will be 51 . The $\mathrm{CW}$ will continue to increase until the maximum size of the window is reached (i.e., $\mathrm{CW}=\mathrm{CW}$ $\max$.

If the size of the contention window needs to be reduced, the $\mathrm{CW}$ is cut in half (i.e., $\mathrm{CW} / 2$ ). If the current $\mathrm{CW}$ is 25 and the window must be decreased, the new value for the $\mathrm{CW}$ is 12. The size of the contention window is able to continue to decrease until the minimum value is reached (i.e., $\mathrm{CW}=\mathrm{CW}$ $\mathrm{min}$ ). The size of the $\mathrm{CW}$ will continuously fluctuate based on the condition of the network. As such, the $\mathrm{CW}$ will either double, be cut in half, or the current window will be maintained. So using this we can reduce the no of collision.

\section{EXPERIMENTAL RESULTS}

As shown in the fig 4, 5 and 6 we change the no of node on the scenario and throughputs, end to end delay and packet loss are calculated and compare between three protocols 802.11 , $802.11 \mathrm{e}$ and ebp. According to the result our propose protocol ebp gives the best result.

\subsection{Throughput}

Throughput is the how much amount of data can be transmitted from one location to other in a given time. In our result we change the no of node on a road and check how much data are transmitted. We compare three protocol and as shown in the fig 4 . When we use our propose protocol as routing protocol will produce better throughput than other one.

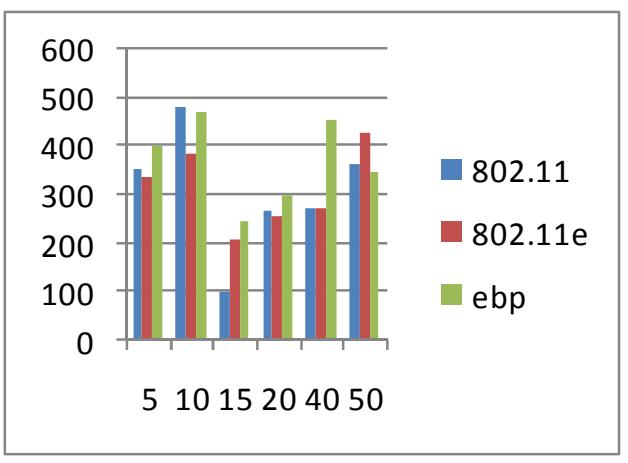

Fig 4 : Throughput by vehicle density

\subsection{End to End Delay}

End to end delay is the time required to transmit the data from one location to other. We also check end to end delay in our work and compare with other one. As shown in the fig 5 using our propose protocol end to end delay is very small.

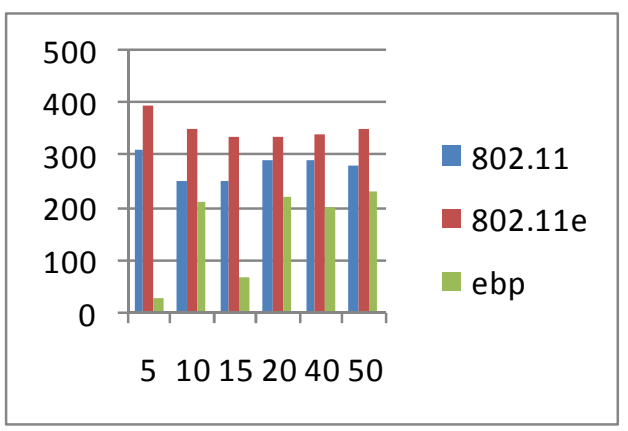

Fig 5: End to end delay by vehicle density

\subsection{Packet Loss}

Packet loss is the no of packet dropped in the communication due to any region like more collision or due to network failure or due to more distance. As we state that our main purpose is to reduce the no of collision in the network and from the fig 6 shows that our protocol gives better result. No of collision using our protocol are very small.

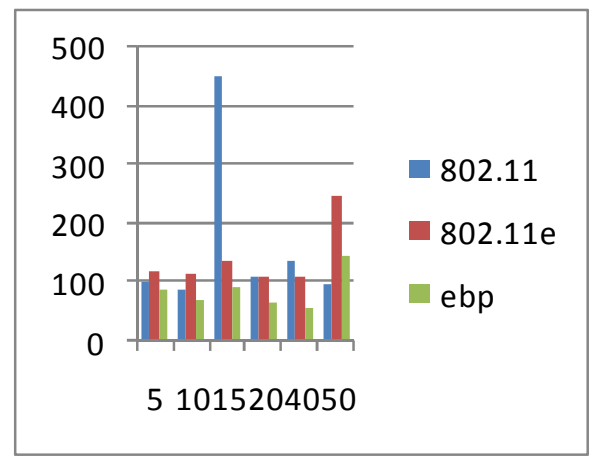

Fig 6: Packet loss by vehicle density

\section{CONCLUSION AND FUTURE WORK}

Dynamically adjusting the contention window improves the reception rate under certain conditions. The results of the simulations show that adaptively adjusting the contention window can increase the reception rate of broadcast frames when there is moderate network traffic. It is unlikely to expect much better results than $802.11 \mathrm{e}$ in the reception rate, under the typical conditions found in a VANET. The proposed additions to the broadcast protocol are not resource intensive or difficult to implement, as a result, implementing the suggested changes provides of increasing the performance of the ebp broadcast protocols for a VANET. In the case of extremely heavy network traffic, the performance of the adaptive broadcast protocol can actually become worse. The reception rate remains approximately the same when the network is saturated with traffic. A number of additional modifications can be made to the 802.11 broadcast protocols to improve the reliability. The following are some areas of future work to improve the performance of the broadcast protocol for a VANET:

Adaptive transmission rate, an algorithm that throttles the rate at which the vehicle's state is transmitted.

Adaptive transmission range, the transmission range can be adjusted to keep the network load on the channel below a certain threshold.

\section{REFERENCES}

[1] Sze-Yao Ni, Yu-Chee Tseng, Yuh-Shyan Chen, and Jang-Ping Sheu. "The broadcast storm problem in a mobile ad hoc network". In MOBICOM, pages 151-162, 1999.

[2] Marc Torrent-Moreno, Daniel Jiang, and Hannes Hartenstein. "Broadcast reception rates and effects of priority access in 802.11-based vehicular ad-hoc networks". In Kenneth P. Laberteaux, Raja Sengupta, Chen-Nee Chuah, and Daniel Jiang, editors, Proceedings of the First International Workshop on Vehicular Ad Hoc Networks, 2004, Philadelphia, PA, USA, October 1, 2004, pages 10-18. ACM, 2004.

[3] Q. Xu, R. Sengupta, and D. Jiang. "Design and analysis of highway safety communication protocol in $5.9 \mathrm{ghz}$ 
dedicated short range communication spectrum”, Spring 2003.

[4] X. Yang, J. Liu, F. Zhao, and N. H. Vaidya. "A vehicleto-vehicle communication protocol for cooperative collision warning". In MobiQuitous, pages 114-123. IEEE Computer Society, 2004.

[5] Pat Jangyodsuk et al. "Reliable Broadcasting in VANET", SJSU 2011.

[6] K. Hua and R. Villafane, "A near-optimal broadcast technique for vehicular networks," 2009 Wireless Telecommunications Symposium, 2009, pp. 1-8.

[7] K. Suriyapaibonwattana and C. Pomavalai, "An Effective Safety Alert Broadcast Algorithm for VANET," 2008 International Symposium on Communications and Information Technologies, 2008, pp. 247-250.

[8] H. Jiang, H. Guo, and L. Chen, "Reliable and Efficient Alarm Message Routing in VANET," 2008 The 28th International Conference on Distributed Computing Systems Workshops, 2008, pp. 186-191.
[9] A. Amoroso, M. Ciaschini, and M. Roccetti, "The farther relay and oracle for VANET. Preliminary results," Proceedings of the 4th Annual International Conference on Wireless Internet, ACM, 2008.

[10] Q. Yang, L. Shen, and W. Xia, "Distributed Probabilistic Broadcasting for Safety Applications in Vehicular Ad Hoc Networks," Wireless Communications \& Signal Processing, 2009. WCSP 2009. International Conference, 2009, pp. 0-4.

[11] O.K. Tonguz, N. Wisitpongphan, J.S. Parikht, F. Bait, P. Mudaliget, and V.K. Sadekart, "On the Broadcast Storm Problem in Ad hoc Wireless Networks," BROADNETS 2006. 3rd International Conference, 2006.

[12] L. Bononi, "A Cross Layered MAC and Clustering Scheme for Efficient Broadcast in VANETs," Mobile Adhoc and Sensor Systems, 2007. MASS 2007. IEEE Internatonal Conference, 2007. 